\title{
Detected and overlooked cervical spine injury among comatose trauma patients: from the Pennsylvania trauma outcomes study
}

\author{
JosePh H. Piatt JR., M.D. \\ Section of Neurosurgery, St. Christopher's Hospital for Children; and Department of Pediatrics, \\ Drexel University College of Medicine, Philadelphia, Pennsylvania
}

\begin{abstract}
Object. A rule for identifying patients with traumatic brain injury (TBI) who are at exceptionally low risk of cervical spine injury might be clinically useful. The goal in this study was to research case records to determine whether such a rule would be practicable.

Methods. The Pennsylvania Trauma Outcomes Study database was used to find patients with TBI in whom Glasgow Coma Scale (GCS) scores at admission were 8 or less. Cases of cervical spine injury were identified from diagnostic codes. Associations between these injuries and a variety of clinical variables were tested using chi-square analysis. The probability of a cervical spine injury in these patients was modeled by logistic regression. Decision tree models were constructed and statistical determinants of overlooked cervical spine injury were examined.

The prevalence of cervical spine injury among 41,142 cases of TBI was $8 \%$. The mechanism of injury, presence of thoracolumbosacral (TLS) spinal, limb and/or facial fracture, patient age, GCS score at admission, and the presence of hypotension were all factors associated with cervical spine injury. These were incorporated into the following logistic regression model: probability of cervical spine injury $=1 /(1+\exp [4.030-0.417 *$ mechanism $-0.264 *$ age $0.678 * \mathrm{TLS}-0.299 * \operatorname{limb}+0.218 * \mathrm{GCS}$ score $-0.231 *$ hypotension $-0.157 *$ facial $]$ ).

This model yielded a rule for clearance of $28 \%$ of cases, with a negative predictive value (NPV) of $97 \%$. Decision tree analysis yielded an easily stated rule for clearance of $24 \%$ of cases, with an NPV of $98.2 \%$. The prevalence of overlooked cervical spine injury among all patients with severe TBI was $0.3 \%$; the prevalence of overlooked cervical spine injury among patients in whom it was later diagnosed was 3.9\%. Overlooked cervical spine injury was less common among patients with associated TLS fractures (odds ratio $0.453,95 \%$ confidence interval $0.245-0.837$ ).

Conclusions. No acceptable rule for relaxation of vigilance in the search for cervical spine injury among patients with severe TBI has been identified. Levels of provider vigilance and consequent rates of overlooked cervical spine injury can be affected by environmental cues and presumably by other behavioral and organizational factors.
\end{abstract}

\section{KEY WORDS - cervical spine injury • coma • decision tree • logistic regression • receiver operating characteristic curve $\bullet$ traumatic brain injury}

Detection and stabilization of cervical spine injury is a major priority in the initial care of trauma victims because of the potentially catastrophic consequences of mismanagement. Patients with concurrent severe TBI are a particular challenge, because they cannot report neck pain and cannot cooperate with neurological examination. Furthermore, mechanisms of injury that transmit sufficient energy to the brain to cause coma necessarily transmit energy to the cervical spine; thus the prevalence of cervical spine injury among patients with traumatic coma is not insignificant. Estimates of the prevalence of this type of injury among pa-

Abbreviations used in this paper: $\mathrm{CI}=$ confidence interval; $\mathrm{GCS}=$ Glasgow Coma Scale; ICD.9.CM = International Classification of Diseases, Ninth Revision; NPV = negative predictive value; OR = odds ratio; PTOS = Pennsylvania Trauma Outcomes Study; ROC = receiver operating characteristic; $\mathrm{TBI}=$ traumatic brain injury; TLS = thoracolumbosacral. tients with traumatic impairment of responsiveness range from to 1.8 to $26 \%$, depending on inclusion criteria. ${ }^{1,3-8,11,13-18}$

Diagnostic investigation of the cervical spine in the comatose patient is a vexing problem. Mere radiography is not sufficiently sensitive to stand alone as the definitive investigation, and radiographs obtained in the setting of acute trauma are often technically unsatisfactory. Computerized tomography scanning of the cervical spine can be performed conveniently along with the initial head scan, but concerns remain about the detection of purely ligamentous injuries and about the possibility of spinal cord injury without a radiographically identified abnormality. ${ }^{5}$ Magnetic resonance imaging can demonstrate ligamentous injuries and intrinsic spinal cord injury, ${ }^{6}$ but the attendant magnetic fields create obstacles to clinical and physiological monitoring. If the initial evaluation of the cervical spine is incomplete, subsequent investigations will necessarily entail 
the inconvenience and risk associated with transporting patients with potentially unstable TBIs between the radiology suite and the intensive care unit. Despite the imperative that every cervical spine injury be detected and managed appropriately, judgment must be exercised, and compromises must be made. Refinement of statistical rules for the assessment of the risk of cervical spine injury among patients with TBI might be useful to the clinician who must set treatment priorities. Specifically, a rule or test with a high NPV might permit deferral or elimination of diagnostic investigations in patients in whom the risk of such an injury is very low.

\section{CLINICAL MATERIAL AND METHODS}

The PTOS is a database incorporating demographic and clinical data for all patients hospitalized at trauma centers accredited by the Pennsylvania Trauma System Foundation. The study group was defined as all patients with admission GCS scores of 8 or less. Selected data pertaining to this study group were requested by the investigator and prepared without identifiers by PTOS staff. The primary dependent variable was cervical spine injury, as defined by ICD.9.CM codes 805.00-805.18; 806.00-806.19; 839.00839.18; and 952.00-952.09, which include cervical spine fracture with or without spinal cord injury, cervical spinal dislocation, and cervical spinal cord injury without fracture or dislocation (open spine injuries were excluded). A secondary dependent variable, overlooked cervical spine injury, was coded separately in the database as a field labeled "discharge diagnosis of cervical spine injury with fracture, subluxation, or neuro deficit not addressed on admission."

Potentially useful independent variables were recoded as necessary in either nominal or ordinal formats: patient age, sex, race, mechanism of injury, place where injury occurred (that is, home/other/road), admission GCS score, associated TLS, limb, and/or facial fractures, the presence of hypotension on admission, level of alcohol intoxication, and payor. Dichotomous nominal variables were assigned values of 0 or 1 if the variable factor was absent or present, respectively. Continuous variables were recoded either as dichotomous nominal variables or as ordinal ones, and nondichotomous nominal variables were recoded as ordinal variables with increasing or decreasing associated prevalences of cervical spine injury (Table 1). Associations between this type of injury and these independent variables were analyzed using cross-tabulation and chi-square tests. For associations between cervical spine injury and dichotomous nominal variables, the ORs were calculated.

The study data set was divided randomly into two equal subsets, a development data set and a validation data set. Backward stepwise likelihood ratio logistic regression was used with the development data set to model the probability of cervical spine injury as a function of independent variables that had exhibited a significant association with this type of injury in the preliminary univariate analysis. The power of the model was then analyzed with the validation data set by using ROC methodology. The utility of the logistic regression model for identification of patients at low risk for cervical spine injury was then analyzed by calculating NPVs at various test probability thresholds, again using the validation data set.

Decision tree models were also created, once again using the nominal and ordinal independent variables associated with cervical spine injury in the preliminary univariate analysis. The Classification and Regression Tree; Quick, Unbiased, Efficient Statistical Tree; and exhaustive ChiSquare Automatic Interaction Detection algorithms were explored in search of simple trees that could be used to sort patients into groups at very low risk for cervical spine injury. The exhaustive Chi-Square Automatic Interaction Detection algorithm allows a node to give rise to more than two branches, so it was judged to mimic clinical thought processes better than the other algorithms. The probability threshold for branching was set at 0.05 , and the Bonferroni correction was used. As for the logistic regression model, trees were created using randomly generated development data sets, and the validity of each tree was checked by analyzing its performance in the complementary validation data set.

\section{Statistical Analysis}

Organization, recoding, and analysis of the data were performed using commercially available software (SPSS Version 11.5 for Windows; SPSS, Inc., Chicago, IL). Decision trees were created and analyzed using AnswerTree Version 3.1 (SPSS, Inc.). This research was approved and supervised by the Institutional Review Board of Drexel University College of Medicine.

\section{RESULTS}

Between October 1986 and August 2004, 41,142 patients with TBI and admission GCS scores less than or equal to 8 were registered in the PTOS. There were 3281 patients whose diagnosis at discharge was cervical spine fracture or dislocation, or cervical spinal cord injury, for a prevalence of $8 \%$ (95\% CI 7.8-8.2\%).

\section{Univariate Associations}

With the exception of alcohol intoxication, all of the independent variables examined had significant associations with cervical spine injury (Table 2). The prevalence of this type of injury increased with age; was higher among female patients; was higher among Caucasians than among African Americans and Asian Americans; was higher among victims of road accidents than among patients with injuries sustained elsewhere; was higher among occupants of motor vehicles, motorcyclists, and pedestrians struck by moving vehicles than among victims of other injury mechanisms; was higher among patients with GCS scores of 3; was higher among patients with associated TLS fractures, limb fractures, or facial fractures; was slightly higher among patients with hypotension; was insignificantly lower among patients with alcohol intoxication; and was higher among patients with "other commercial" insurance coverage.

Because the sample size was so large, several associations of minor or uncertain clinical significance reached high levels of statistical significance. The association of payor with cervical spine injury was particularly dubious. This variable was examined as a surrogate for socioeconomic status, other data reflecting this status having been expunged in the deidentification process. For purposes of analysis the original 12 payor categories were sorted according to associated prevalences of cervical spine injury 
TABLE 1

Coding of independent variables in patients who were comatose after trauma*

\begin{tabular}{|c|c|c|c|c|c|}
\hline Variable & \multicolumn{5}{|c|}{ Category \& Value } \\
\hline injury mechanism & other & bicycle accident & fall & $\begin{array}{l}\text { motor vehicle (driver or } \\
\text { passenger), motorcycle, } \\
\text { or pedestrian accident }\end{array}$ & - \\
\hline age group (yrs) & $0-15$ & $16-30$ & $31-50$ & $51-69$ & $70+$ \\
\hline place injury occurred & home & other & road & - & - \\
\hline insurer & $\begin{array}{l}\text { HMO, PPO, Champus, } \\
\text { Champva, all Medicaid, } \\
\text { self-pay }\end{array}$ & $\mathrm{BC} / \mathrm{BS}$ & $\begin{array}{l}\text { government, workers' } \\
\text { comp, all Medicare }\end{array}$ & other commercial & - \\
\hline $\begin{array}{l}\text { hypotension (SBP } \\
\text { on admission) }\end{array}$ & no hypotension & $\begin{array}{l}<70 \mathrm{~mm} \mathrm{Hg} \text {, ages } 0-15 \mathrm{yrs} \\
\quad<80 \mathrm{~mm} \mathrm{Hg}, 16-69 \mathrm{yrs} \\
<90 \mathrm{~mm} \mathrm{Hg}, 70+\text { yrs }\end{array}$ & - & - & - \\
\hline $\begin{array}{l}\text { alcohol intoxication } \\
(\mathrm{g} / \mathrm{dl})\end{array}$ & $<100$ & $>100$ & - & - & - \\
\hline
\end{tabular}

$* \mathrm{BC} / \mathrm{BS}=\mathrm{Blue}$ Cross/Blue Shield; comp = compensation; $\mathrm{HMO}=$ Health Maintenance Organization; $\mathrm{PPO}=$ preferred provider organization; $\mathrm{SBP}=$ systolic blood pressure; $-=$ coding did not extend to the number designated

and lumped into four larger categories, but the larger categories had unintuitive compositions: "self-pay" fell into the same category as "PPO" (preferred provider organization), and "Blue Cross/Blue Shield" was associated with a much lower prevalence of cervical spine injury than "other commercial" insurers (7.8\% compared with $11.5 \%)$. Interestingly, the same associations between payor categories and prevalence of cervical spine injury persisted after random partition of the entire data set into development and validation data sets, as described earlier. Even though payor seemed robust as a predictor of the risk of cervical spine injury, its clinical meaning and usefulness were unclear. The payor variable was therefore suppressed in subsequent model development.

\section{Logistic Regression}

The entire data set was divided randomly into development and validation data sets of equal size. Logistic regression was performed on the development data set to create a

TABLE 2

Independent variables associated with cervical spine injury

\begin{tabular}{lcc}
\hline \hline \multicolumn{1}{c}{ Variable } & $\begin{array}{c}\text { Chi-Square } \\
\text { p Value }\end{array}$ & OR (95\% CI) \\
\hline age & $<0.0005$ & - \\
sex & $<0.0005$ & $1.294(1.197-1.399)$ \\
race & $<0.0005$ & - \\
injury mechanism & $<0.0005$ & - \\
place of injury & $<0.0005$ & - \\
admission GCS score & $<0.0005$ & $0.745(0.685-0.811)$ \\
fractures & & \\
$\quad$ TLS spine & $<0.0005$ & $2.760(2.526-3.016)$ \\
$\quad$ long bone & $<0.0005$ & $2.062(1.916-2.218)$ \\
$\quad$ facial & $<0.0005$ & $1.341(1.235-1.457)$ \\
hypotension & 0.008 & $1.115(1.028-1.208)$ \\
alcohol intoxication & 0.31 & $0.954(0.873-1.043)$ \\
payor & $<0.0005$ & - \\
\hline
\end{tabular}

$*-=$ ORs cannot be calculated. model for identifying cervical spine injury by using a backward stepwise likelihood ratio method and the following independent variables: age, sex, race, injury mechanism, place where injury occurred, GCS score, TLS fracture, limb fracture, facial fracture, hypotension, and intoxication. Sex, race, place, and intoxication were rejected from the model, leaving the following formula: probability $=1$ / $(1+\exp [4.030-0.417 *$ mechanism $-0.264 *$ age $0.678 * \mathrm{TLS}-0.299 * \mathrm{limb}+0.218 * \mathrm{GCS}$ score $0.231 *$ hypotension $-0.157 *$ facial $]$ ).

The logistic regression model was tested in the validation data set by using ROC methodology (Fig. 1). The area under the curve of the ROC (that is, the probability that a randomly selected patient with cervical spine injury has a higher model probability of this type of injury than a randomly selected patient without the injury) was 0.673 (95\% CI 0.657-0.688).

Table 3 shows the performance of the model in the validation data set if it was used as a rule for dismissing the possibility of cervical spine injury, that is, for "clearance" of the cervical spine. Patients in whom the model predicted a probability of this type of injury below an arbitrary threshold might be considered out of danger. As the probability threshold for clearance of the cervical spine is lowered, the NPV, which is the assurance of the absence of cervical spine injury, increases. As the threshold is lowered, however, the fraction of the total population that can be cleared decreases, and the rule becomes less useful.

\section{Decision Trees}

Decision trees were constructed to sort cases according to the presence or absence of cervical spine injury, based on the following variables: age, sex, race, injury mechanism, place where injury occurred (home/other/road), GCS score, TLS fracture, limb fracture, facial fracture, hypotension, and alcohol intoxication. With respect to the goal of finding a simple rule to identify patients at very low risk of cervical spine injury, trees of five, six, and seven layers did not perform any better than the four-layer tree in Fig. 2. A 


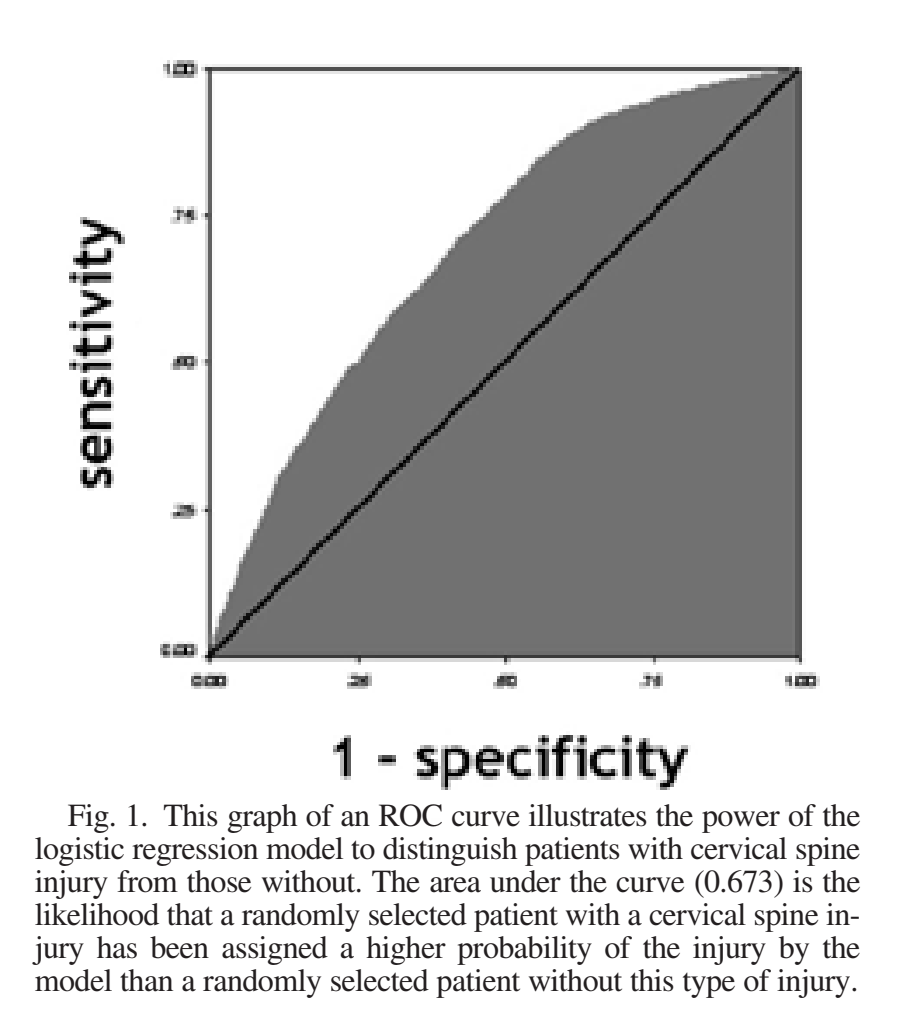

sequence of four branches (mechanism $=$ other, TLS fracture $=$ absent, limb fracture $=$ absent, and facial fracture $=$ absent) yielded a subgroup of the development data set composed of $23 \%$ of cases with a $1.7 \%$ risk of cervical spine injury. In the validation data set, this subgroup constituted $23.7 \%$ of cases, and the risk of injury was $1.8 \%$. The corresponding NPV was therefore $98.2 \%$.

\section{Overlooked Cervical Spine Injury}

Systematic recording of overlooked cervical spine injury did not begin until 1991, and there were entries in this field for 26,936 cases. Consistency between recording of overlooked cervical spine injury and recording of ICD.9.CM diagnoses was not perfect. Of 159 cases coded as overlooked cervical spine injury, there were no corresponding ICD.9.CM diagnoses for $66(42 \%)$. The nature of the injuries that were overlooked in these instances could not be determined. Inspection of the ICD.9.CM diagnoses in the discrepant cases yielded no consistent patterns. Only cases of overlooked cervical spine injury with corresponding ICD.9.CM diagnoses were analyzed further.

The overall rate of overlooked cervical spine injury among all patients with severe TBI was $0.3 \%$. Among 2414 patients with injury to the cervical spine for whom data were available, the rate of overlooked injury was 3.9\%. Overlooked injuries were compared with other cases of cervical spine injury by cross-tabulation and chi-square testing with respect to the following independent variables: age, sex, race, injury mechanism, place where injury occurred, GCS score, TLS fracture, limb fracture, facial fracture, hypotension, alcohol intoxication, and insurance carrier. The only association that attained significance was TLS fracture $(\mathrm{p}=$
TABLE 3

Performance of the logistic regression model as a rule for "clearance" of the cervical spine*

\begin{tabular}{|c|c|c|}
\hline Threshold for Predicting CSI & NPV & Patients "Cleared" $(\%)$ \\
\hline \multicolumn{3}{|l|}{ probability of CSI } \\
\hline$\geq 0.5$ & 0.913 & 100 \\
\hline$\geq 0.25$ & 0.915 & 99 \\
\hline$\geq 0.1$ & 0.939 & 68 \\
\hline$\geq 0.05$ & 0.970 & 28 \\
\hline$\geq 0.025$ & 0.975 & 10 \\
\hline
\end{tabular}

* CSI = cervical spine injury

0.009, Fisher's exact test); a TLS fracture was associated with a reduced risk of overlooked cervical spine injury (OR $0.453,95 \%$ CI $0.245-0.837$ ).

Detection of cervical spine injury at the time of admission seemed to improve during the years covered by the registry. The rates of overlooked occurrences as a fraction of all cervical spine injuries were $10.5,10.2$, and $8.4 \%$ in 1991, 1992, and 1993, respectively, but in subsequent years the fraction of cervical spine injuries that were overlooked stabilized at much lower levels (Fig. 3). From 1994 through 2003 , the mean rate of overlooked cervical spine injury was only $2.3 \%$. Logistic regression of overlooked injuries of this type as a function of registry year was highly significant over the 1991 through 2003 epoch $(p<0.0005)$, but it did not achieve significance over the 1994 through 2003 epoch $(p=0.053)$. (Linear regression was not used for this analysis because the assumptions required for calculation of statistical significance were not valid.) A robust protective effect of concomitant TLS fracture against overlooked cervical spine injury persisted in the 1994 through 2003 epoch, however $(\mathrm{p}=0.028$, Fisher exact test; OR 0.412 , 95\% CI 0.186-0.916).

\section{DISCUSSION}

Both the strengths and the weaknesses of this study derive from the source of its data, a statewide trauma registry. The data set was collected prospectively, and it reflects actual practices at centers dedicated to trauma care that were nonetheless presumably not devoting more attention to cervical spine assessment than to other urgent clinical matters of comparable importance. The data set was also quite large. The $8 \%$ prevalence of cervical spine injury among patients with severe TBI reported here is based on a denominator that is larger, by more than an order of magnitude, than any other comparable study group previously reported. On the other hand, although the data were collected by trained program coordinators, there was no auditing, so no quantitative judgment can be made about accuracy, nor can inconsistencies in the recording of data be clarified retrospectively.

The goal of this investigation was to formulate a simple, practical rule for identifying patients with severe TBI at sufficiently low risk of cervical spine injury that definitive imaging investigations might be safely deferred or dismissed. Because the prevalence of cervical spine injury among the patients with TBI was only $8 \%$, a rule with a sufficiently high NPV might offer great savings in effort and 
resources if it were applicable to a large enough portion of the $92 \%$ of patients without this type of injury. As expected, in this study there was an inverse relationship between the NPVs of the rules examined and their ranges of applicability, and therefore compromises were necessary. With the cervical spine injury probability threshold set at 0.05 , the logistic regression rule was applicable to $28 \%$ of cases and yielded an NPV of 97\%. Lowering the threshold further yielded a higher NPV at the expense of a narrower range of applicability (Table 3). The decision tree rule was similarly applicable to $24 \%$ of cases and yielded an NPV of $98.2 \%$. Unlike logistic regression, decision trees yield rules that are relatively simple to state, in this case as follows: "comatose trauma victims who were not injured by a motor vehicle, in a fall, or on a bicycle, and who do not have facial, limb, or other spinal fractures are at low risk of CSI [cervical spine injury]." (This general statement is consistent with the widely held perception that victims of isolated cranial penetrating trauma are at negligible risk of cervical spine injury..$^{9,10,12}$ )

Rational definition of "sufficiently low risk" is a task that requires a formal cost/benefit analysis, which was outside the scope of this undertaking. ${ }^{2}$ Indeed, the contemporary practice environment does not tolerate any risk of overlooked cervical spine injury. One logical quantitative benchmark, however, is the rate of this type of injury that is over-

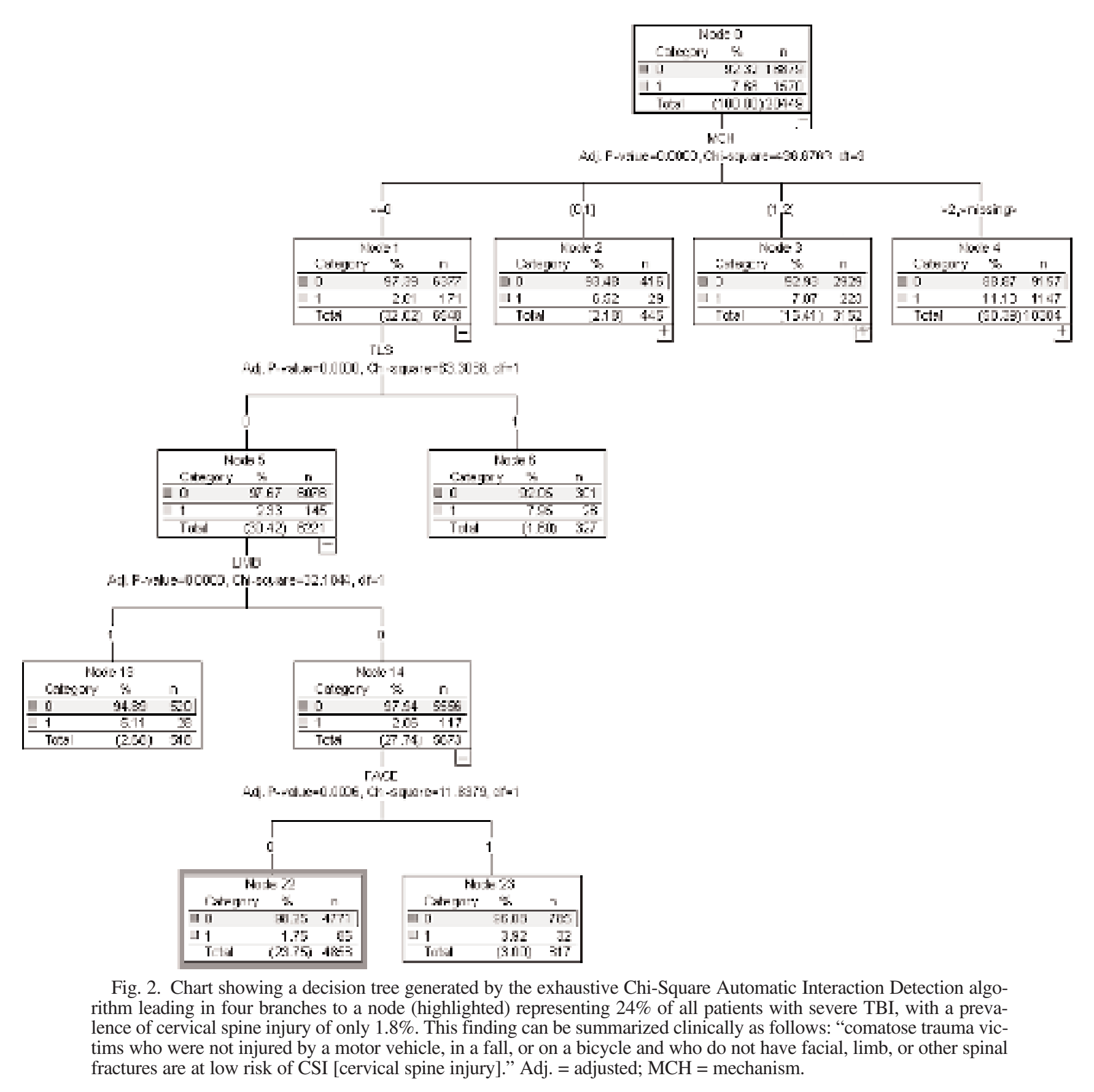




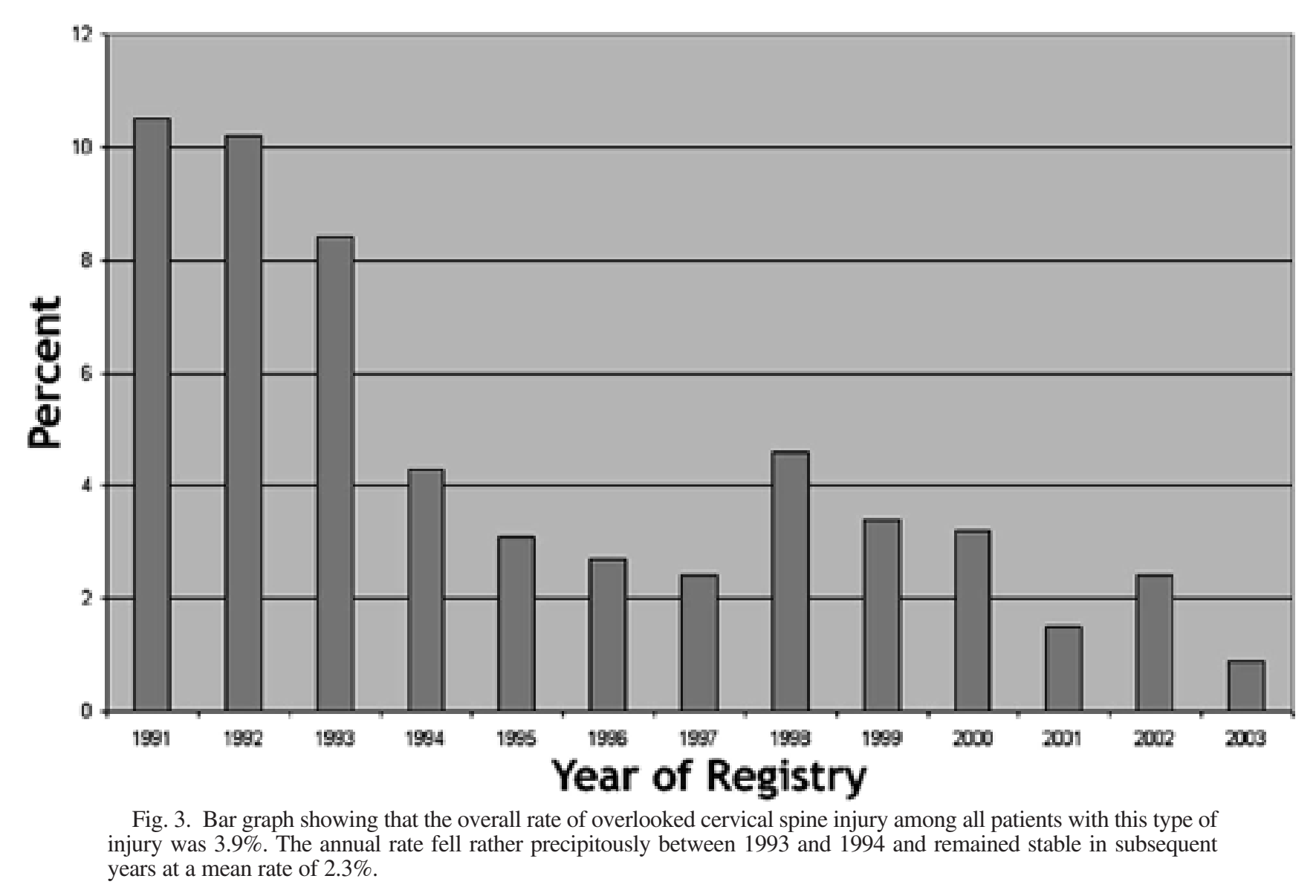

looked by customary diagnostic vigilance at the time of admission. In this study, cervical spine injury was overlooked in $3.9 \%$ of patients in whom it was later found, or in $0.3 \%$ of all patients with severe TBI; thus, customary diagnostic vigilance might be said to have an NPV of $99.7 \%$. A clinical decision rule for clearance of the cervical spine that performs as well on a defined subset of cases as the customary diagnostic investigation performs on unselected patients would by definition save resources without diminishing safety. Neither the logistic regression methodology (NPV 97\%) nor the decision tree methodology (NPV 98.2\%) reached this benchmark. Although its simplicity may be attractive, implementation of the decision tree rule theoretically subjects patients with TBI to a sixfold greater risk of overlooked cervical spine injury than do customary diagnostic practices. Few clinicians would judge such a rule to be suitable for incorporation into clinical guidelines or routine practice.

Injury at one level of the spine increases the likelihood of injury at other levels. The prevalence of cervical spine injury among patients with severe TBI was $8 \%$ in this study, but its prevalence among patients with TBI who also had suffered TLS fractures was $23 \%$. Physicians who provide trauma care are evidently aware of this association, and awareness affects behavior. In this study, the presence of TLS spinal fractures significantly lowered the risk of overlooked cervical spine injury, from 4.4 to $2.1 \%$. Once providers identified an injury at one level, they seemed to look harder for injuries at other levels. This observation indi- cates that the overall rate of overlooked injury in this study, $3.9 \%$, is not an irreducible product of the inadequacy of imaging technology and the distractions of the trauma bay. It is a function of the motivational state of the provider and can be affected by environmental cues such as TLS spine injury, and presumably by organizational improvements in the system of care delivery.

Detection of cervical spine injury did indeed improve during the years recorded in the PTOS registry. After 3 early years of high rates of overlooked injuries of this type, the rates fell rather dramatically in 1994 and remained stable and low thereafter. The explanation for this favorable development is purely a matter of speculation. Increasing standardization of care, more liberal use of computerized tomography scanning of the cervical spine, and a systematic change in the data entry practices of the local program coordinators are all possibilities. Even if deliberate quality improvement efforts deserve credit, the data regarding associated TLS spinal fractures show that room for additional improvement remains. The protective effect of associated TLS fracture persisted unchanged in 1994 and in subsequent years, demonstrating that enhanced provider vigilance, whether achieved by recognition of related injuries or by other means, can lower rates of overlooked cervical spine injury still further.

\section{CONCLUSIONS}

In contemporary standards of care, overlooked cervical 
spine injury is not tolerated. Among comatose patients with TBI in the large data set presented here, the overall risk of overlooked cervical spine injuries in the face of customary diagnostic vigilance was $0.3 \%$. This exercise in statistical modeling failed to produce a simple rule that permits deferral or dismissal of diagnostic investigations for any significant fraction of patients with TBI in whom the risk of overlooked cervical spine injuries is comparably low. The protective effect of associated TLS fractures on the risk of overlooked cervical spine injury indicates that certain environmental cues serve to enhance provider vigilance. Engineered modifications of the clinical environment that similarly enhance vigilance may further reduce the risk of overlooked cervical spine injury.

\section{References}

1. Bayless P, Ray VG: Incidence of cervical spine injuries in association with blunt head trauma. Am J Emerg Med 7:139-142, 1989

2. Blackmore CC, Ramsey SD, Mann FA, et al: Cervical spine screening with CT in trauma patients: a cost-effectiveness analysis. Radiology 212:117-125, 1999

3. Brohi K, Healy M, Fotheringham T, et al: Helical computed tomographic scanning for the evaluation of the cervical spine in the unconscious, intubated trauma patient. J Trauma 58:897-901, 2005

4. Brooks RA, Willett KM: Evaluation of the Oxford protocol for total spinal clearance in the unconscious trauma patient. $\mathbf{J}$ Trauma 50:862-867, 2001

5. Chiu WC, Haan JM, Cushing BM, et al: Ligamentous injuries of the cervical spine in unreliable blunt trauma patients: incidence, evaluation, and outcome. J Trauma 50:457-464, 2001

6. D'Alise MD, Benzel EC, Hart BL: Magnetic resonance imaging evaluation of the cervical spine in the comatose or obtunded trauma patient. J Neurosurg 91 (Suppl 1):54-59, 1999

7. Hills MW, Deane SA: Head injury and facial injury: is there an increased risk of cervical spine injury? J Trauma 34:549-554, 1993

8. Holly LT, Kelly DF, Counelis GJ, et al: Cervical spine trauma associated with moderate and severe head injury: incidence, risk factors, and injury characteristics. J Neurosurg 96 (Suppl 3):285-291, 2002

9. Kaups KL, Davis JW: Patients with gunshot wounds to the head do not require cervical spine immobilization and evaluation. J Trauma 44:865-867, 1998

10. Kennedy FR, Gonzalez P, Beitler A, et al: Incidence of cervical spine injury in patients with gunshot wounds to the head. South Med J 87:621-623, 1994

11. Laham JL, Cotcamp DH, Gibbons PA, et al: Isolated head injuries versus multiple trauma in pediatric patients: do the same indications for cervical spine evaluation apply? Pediatr Neurosurg 21:221-226, 1994

12. Lanoix R, Gupta R, Leak L, et al: C-spine injury associated with gunshot wounds to the head: retrospective study and literature review. J Trauma 49:860-863, 2000

13. Michael DB, Guyot DR, Darmody WR: Coincidence of head and cervical spine injury. J Neurotrauma 6:177-189, 1989

14. O'Malley KF, Ross SE: The incidence of injury to the cervical spine in patients with craniocerebral injury. J Trauma 28: 1476-1478, 1988

15. Ross SE, O'Malley KF, DeLong WG, et al: Clinical predictors of unstable cervical spinal injury in multiply injured patients. Injury 23:317-319, 1992

16. Schenarts PJ, Diaz J, Kaiser C, et al: Prospective comparison of admission computed tomographic scan and plain films of the upper cervical spine in trauma patients with altered mental status. J Trauma 51:663-668, 2001

17. Soicher E, Demetriades D: Cervical spine injuries in patients with head injuries. Br J Surg 78:1013-1014, 1991

18. Widder S, Doig C, Burrowes P, et al: Prospective evaluation of computed tomographic scanning for the spinal clearance of obtunded trauma patients: preliminary results. J Trauma 56: 1179-1184, 2004

Manuscript received August 15, 2005.

Accepted in final form September 9, 2005.

Address reprint requests to: Joseph H. Piatt Jr., M.D., Section of Neurosurgery, St. Christopher's Hospital for Children, Erie Avenue at Front Street, Philadelphia, Pennsylvania 19134-1095. email: joseph. piatt@tenethealth.com. 